\title{
VOLUME 28: STATISTICS AND THANKS
}

In its 28th year of publication, the International Journal of Technology Assessment in Health Care has once again enjoyed active input from authors, reviewers, and the Editorial Board. The meeting of the Editorial Board in Bilbao discussed journal processes and principles, agreeing that reviewers benefit from receiving feedback about the process and that the reference system can be changed. Reviewers can now view the decision letters and author responses through the review system.

From this volume, the Journal will apply the Uniform Requirements for Manuscripts Submitted to Biomedical Journals. This means references from now on are numbered consecutively in order of appearance in the text, cited in text by Arabic numerals in parentheses. Details are available at: http://www.nlm.nih.gov/bsd/uniform_requirements.html and in the Journal inside cover.

For volume 28, this Journal received 169 original manuscripts and 2 letters. From September 2011 to August 2012, these manuscripts were sent from thirty-five different countries; 47 percent of the first authors were from Europe and 19 percent from North America. This indicates a slight but continuing increase of submissions from other parts of the world. By September 2012, sixty-seven papers and two letters (36 percent of submissions) had been accepted for publication. The impact factor increased to 1,365 .

The 287 authors of sixty-nine published papers represented all continents. First authors were most often from the United Kingdom (17 percent) or Canada (14 percent), and 62 percent of first authors were from Europe. The proportion of methodological papers ( 22 percent) and policy studies ( 15 percent) were similar to previous years. A methodological article was accompanied by three invited comments, and a mini-theme on Disinvestment presented three original papers. The theme section on "Early awareness and alert methods and systems" consisted of seven peer-reviewed papers, invited by the theme editors whom the Journal thanks warmly for their time and expertise.

A good peer reviewer is swift, thorough, and polite, formulating clear comments for authors on how to improve on the manuscript. This Journal's additional requirement is a broad understanding of many aspects of HTA. For volume 28, we received 286 reviews from 194 reviewers. From among them, the 2012 Best Reviewer title goes to Irina Cleemput from Belgium. Her special expertise in economic evaluation and health policy has been much valued by IJTAHC authors and editors for many years, so congratulations are deserved. The Journal is grateful for the input of the following persons who have reviewed manuscripts in the past year:

Matthew Allsop, Heidi Anttila, Toni Ashton, Christel Bahtsevani, David Banta, Jeremiah Barondess, Christian Becker, Christine Bergeron, Juan Antonio Blasco, Andrew Booth, Neill Booth, Andrew Boyd, Lundy Braun, Elisabeth Breyer, John Bridges, Eduardo Briones, James Brophy, Reinhard Busse, Javier Caballero-Villarraso, Daniel Callejo, Alun Cameron, Bruce Campbell, Thane Chambers, Rong-Rong Chen, Jody Church, Americo Cicchetti, Irina Cleemput, Andy Clegg, Fiona Clement, Annalijn Conklin, Michael Craven, Peter Csonka, Derek Cutler, Tim Dall, Thomas Davidson, Karin Denton, Francois Dionne, Carmen Dirksen, Persephone Doupi, Karla Douw, Adam Elshaug, Karen Facey, David Feeny, Harriet FinneSoveri, Signe Flottorp, Atle Fretheim, Anne Fry-Smith, Katrine Frønsdal, Marie-Pierre Gagnon, Giselle Gallego, Pedro Gallo, Renato Garcia, Sarah Garner, Lou Garrison, Mats Geijer, Christian Gericke, Alan Girling, Ron Goeree, Stephen Goodall, Clifford Goodman, Vicki Goodwin, Javier Gracia, Alicia Granados, Roberto Grilli, Kees Groeneveld, Janneke Grutters, Iñaki Gutierrez-Ibarluzea, David Hailey, Ariel Hammerman, Dorte Gilså Hansen, Helle Hansen, Ingrid Harboe, Heli Hatonen, Chris Henshall, Janet Hiller, Hannele Hyppönen, Maarten IJzerman, Mohammed Jabr, Tiina Jahkola, Don Juzwishin, Bengt Jönsson, Ingvar Karlberg, Brendon Kearney, Ilmo Keskimäki, Kristian Kidholm, Lars Klack, Marianne Klemp, Gisele Kobelt, Katarzyna Kolasa, Heli Kotilainen, Finn Kristensen, Ivar Kristiansen, Tanja Krones, Ilkka Kunnamo, Hirohiko Kuratsune, Giuseppe La Torre, Jorma Lahtela, Kristian Lampe, Vigdis Lauvrak, Frédéric Lavoie, Marjut Leidenius, Jaana Leipälä, Yun Li, Morgan Lim, David Low, Georgios Lyratzopoulos, Esa Läärä, Guy Maddern, Sumit R. Majumdar, Nea Malila, Timo Maljanen, Antti Malmivaara, Deborah Marshall, Jennifer Martin, Josephine Mauskopf, Brendan McIntosh, Tracy Merlin, Ruairidh Milne, Barbara Mintzes, Mowatt, Graham, Debjani Mueller, Matti Mäkelä, Suvi Mäklin, Berit Mørland, Anna Nachtnebel, Mona Nasser, Aljoscha Neubauer, Duncan Neuhauser, Rita Nevesdefaria, Tom Noseworthy, Vitalis Nwagbara, Arto Ohinmaa, Daniela Oliveira, Sari Ormstad, Andrew D. Oxman, Claire Packer, Dimitra Panteli, Iris Pasternack, Steve Pearson, Matthias Perleth, Paulo Picon, Julie Polisena, Keng Ho Pwee, Jasmine Pwu, Feng Qian, James Raftery, Ulla-Maija 
Rautakorpi, Eeva Reissell, Rosa Rico, Karen Ritchie, Risto P. Roine, Ingrid Rosian-Schikuta, John-Arne Rottingen, Alberto Ruano-Ravina, Alric Rüther, Pirjo Räsänen, Maija Saijonkari, Laura Sampietro-Colom, Rahul Sasane, Petra SchnellInderst, Ruth Schwarzer, Ann Scott, Joshua Shemer, Sue Simpson, Becky Skidmore, Tania Stafinski, Andrew Stevens, Jackie Street, Heidi Stürzlinger, Hae Sun Suh, Tarja Suominen, Claudette Sutherland, Rosanna Tarricone, Rod Taylor, Brenda Tong, Leigh-Ann Topfer, Leena Tuomisto, Tarja Vainiola, Gert Jan van der Wilt, Giedrius Vanagas, Leonor Varela-Lema, Adriana Velazquez-Berumen, Hindrik Vondeling, Catherine Voutier, Bert Vrijhoef, Olli Väisänen, Janet Wale, Sophie Werko, Claudia Wild, Torbjørn Wisløff, Durhane Wong-Rieger, Wenya Yang, and Ingrid Zechmeister.
The Editorial Office continues to welcome input from authors and readers at IJTAHC@thl.fi.

Marjukka Mäkelä

FINOHTA (Finnish Office for Health Technology Assessment) At THL (National Institute for Health and Welfare)

PO Box 30, 00271 Helsinki, Finland

Terhi Ilonen

FINOHTA (Finnish Office for Health Technology Assessment) At THL (National Institute for Health and Welfare)

PO Box 30, 00271 Helsinki, Finland 